OPEN ACCESS

Edited by:

Weihong Jiang,

Central South University, China

Reviewed by:

Zhouying Peng,

Central South University, China

A. B. Zulkiflee

University Malaya Medical

Centre, Malaysia

*Correspondence:

Bin Huang

xgwhb@126.com

Xiao Rong Wen

hxwenxiaor@126.com

tThese authors have contributed equally to this work and share first

authorship

Specialty section:

This article was submitted to

Otorhinolaryngology - Head and Neck

Surgery,

a section of the journal

Frontiers in Surgery

Received: 01 June 2021

Accepted: 04 August 2021

Published: 27 September 2021

Citation:

Chen GX, Chen CW, Wen XR and Huang B (2021) Intravascular Fasciitis

of the Jugular Vein Mimicking

Thrombosis and Sarcoma: A Case

Report. Front. Surg. 8:715249.

doi: 10.3389/fsurg.2021.715249

\section{Intravascular Fasciitis of the Jugular Vein Mimicking Thrombosis and Sarcoma: A Case Report}

\author{
Guo Xin Chen ${ }^{1+}$, Chu Wen Chen ${ }^{1+}$, Xiao Rong Wen ${ }^{2 *}$ and Bin Huang ${ }^{1 *}$ \\ ${ }^{1}$ Department of Vascular Surgery, West China Hospital, Sichuan University, Chengdu, China, ${ }^{2}$ Department of Ultrasound \\ Medicine, West China Hospital, Sichuan University, Chengdu, China
}

Background: Intravascular fasciitis is a rare disease that is a reactive proliferative lesion of myofibroblasts. There are rare reports that intravascular fasciitis has invaded the jugular vein as seen in this case.

Case Presentation: A 41-year-old female presented with right neck dull pain for 20 days. The appearance of the subcutaneous mass was oval, pink hyaline, well-demarcated, and measuring $\sim 5 \mathrm{~mm}$ in diameter. Microscopically, the mass was composed of spindle cells arranged in intersecting fascicles. Immunohistochemical stains showed that the spindle cells were positive for smooth muscle actin and negative for S-100, Desmin, MyoD1, and elastin stains. The nuclei of the spindle cells were relatively uniform, and mitotic activity was observed. The overall morphological and immunohistochemical features are consistent with intravascular fasciitis.

Conclusion: Due to the rapid growth and vascular invasion, intravascular fasciitis created a high risk of misdiagnosing it as a sarcoma or thrombosis. Reporting this uncommon case, we raise awareness of this non-neoplastic lesion, and careful, light microscopic examination combined with immunohistochemical staining aids in the diagnosis of intravascular fasciitis.

Keywords: intravascular fasciitis, internal jugular vein, thrombosis, sarcoma, surgery

\section{INTRODUCTION}

Intravascular fasciitis (IVF) is a rare benign lesion arising from reactive proliferation of myofibroblasts inside the vascular lumen or in the superficial or deep fascia with involvement of small- and/or medium-size blood vessels. IVF often occurs in the upper extremities, head and neck, and lower extremities. Five cases involving the large vein are previously reported in the literature and are commonly misdiagnosed as venous thrombosis or malignant sarcoma. Here, we report a rare case of IVF that was located in the right internal jugular vein. Meanwhile, we review the literature on this rare disease.

\section{CASE PRESENTATION}

A 41-year-old female presented with right neck dull pain for 20 days. She denied any associated difficulty with headache, dizziness, pain, fever, or swelling. She was a nonsmoker and non-drinker and denied any drug abuse. Physical examination revealed a firm, well-defined, tender, non-mobile 


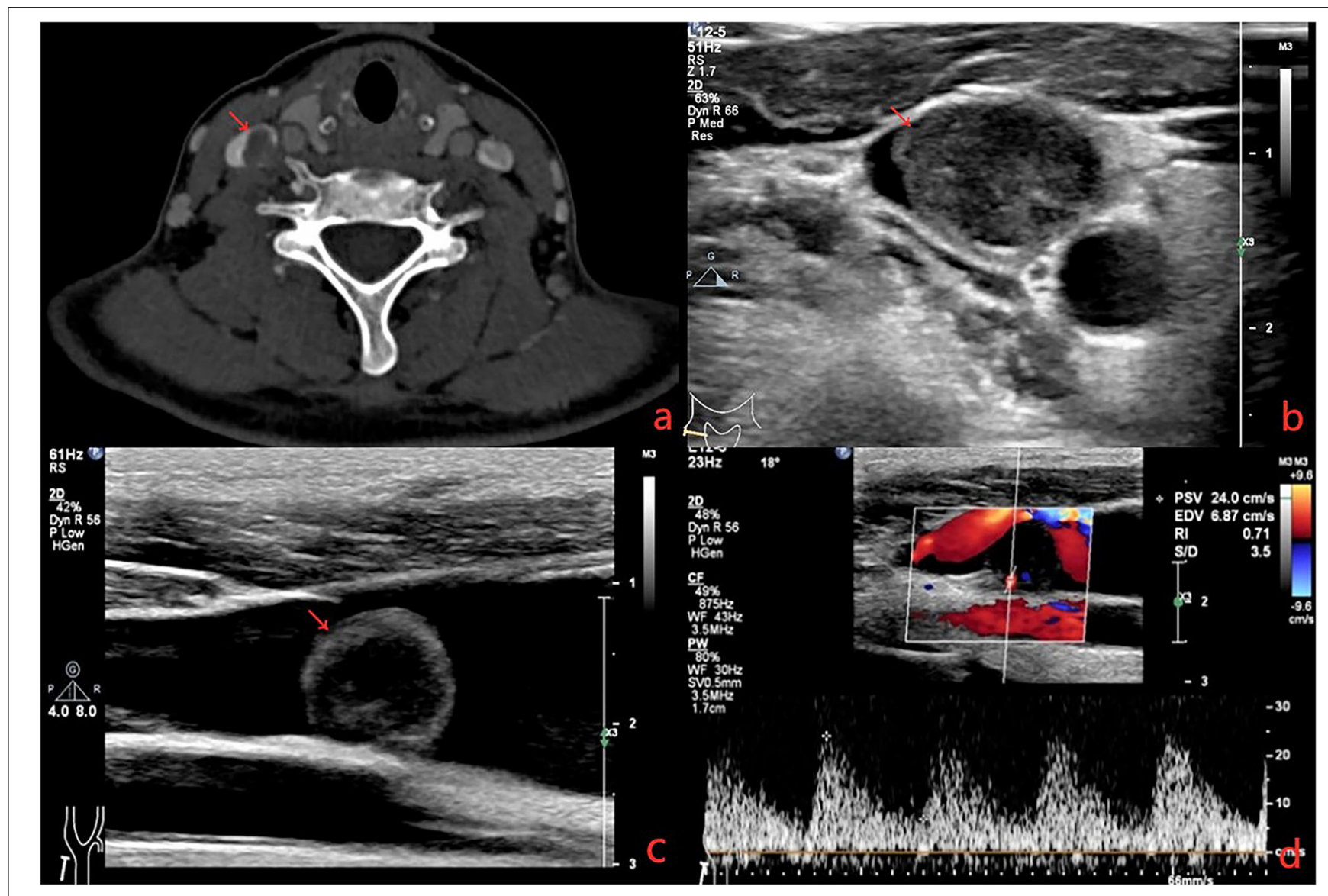

FIGURE 1 | (a) Computerized tomography shows a solid, non-enhanced nodule in the right internal jugular vein (RJV) (red arrow: nodule, green arrow: RJV). (b) Ultrasound shows the nodule arising from the RJV without extravascular invasion (red arrow: nodule, green arrow: RJV). (c) Ultrasound shows the nodule led to severe RJV stenosis (red arrow: nodule, green arrow: RJV, red triangle: right internal carotid artery). (d) Doppler ultrasound detected the blood flow signals in the nodule.

mass in the right neck with dimension about $10 \times 10 \times 10 \mathrm{~mm}$. The mass did not move up or down with swallowing. Laboratory data were within normal limits. A computed tomography angiography (CTA) demonstrated the right jugular vein filling defect at the level of the cricoid cartilage-thyroid plane (Figure 1a). The grayscale ultrasound and color Doppler flow imaging showed the right internal jugular vein had a hypoechoic mass about $10 \times 11 \times 11 \mathrm{~mm}$ with a clear boundary, and the mass had a 3.9-millimeter-wide base located in the posterior wall of the internal jugular vein with a linear blood flow signal in this mass (Figures 1b,c). The pulse Doppler ultrasonography detected arterial spectrum in the mass with the peak systolic blood flow velocity: $24 \mathrm{~cm} / \mathrm{s}$, the end-diastolic blood flow velocity: $6.87 \mathrm{~cm} / \mathrm{s}$, and the resistance index: 0.71 (Figure 1d). The ultrasound indicated neoplastic lesions originating from the internal jugular vein (Figures $\mathbf{1 b}, \mathbf{c}$ ). After medical clearance, the patient received complete surgical excision with the internal jugular vein reconstructed under monitored anesthesia care. In the operation, the mass filled the entire lumen of the internal jugular vein, and compensated distention of the proximal part of the internal jugular vein was observed. The specimen excised was a $13 \times 10 \times 18 \mathrm{~mm}$ mass without extravascular invasion and involvement of any surrounding structures (Figure 2).

Histopathological investigation revealed that a wellcircumscribed nodular growth of fibroblasts and myofibroblasts in a collagenous stroma in large areas and nuclear fission was evident. Immunohistochemically, the cells in the lesion and the blood vessel wall were diffusely positive for smooth muscle actin (SMA). S100, Desmin, MyoD1, and elastin stains were negative. Fluorescent in situ hybridization (FISH) showing rearrangement of the USP6 locus (separation of green and red signals) and excluding gene amplification of the MDM2 (Figure 3). Our research protocol was approved by the local ethics committee.

\section{DISCUSSION}

Nodular fasciitis (NF) is a benign lesion arising in the subcutaneous tissue, skeletal muscles, and fascia. IVF is a rare variant of NF, which was first described by Patchefsky and Enzinger in 1981 (1), accounting for $<3 \%$ of NF (2). In total, 38 patients with IVF are reported in cases recorded in PubMed 


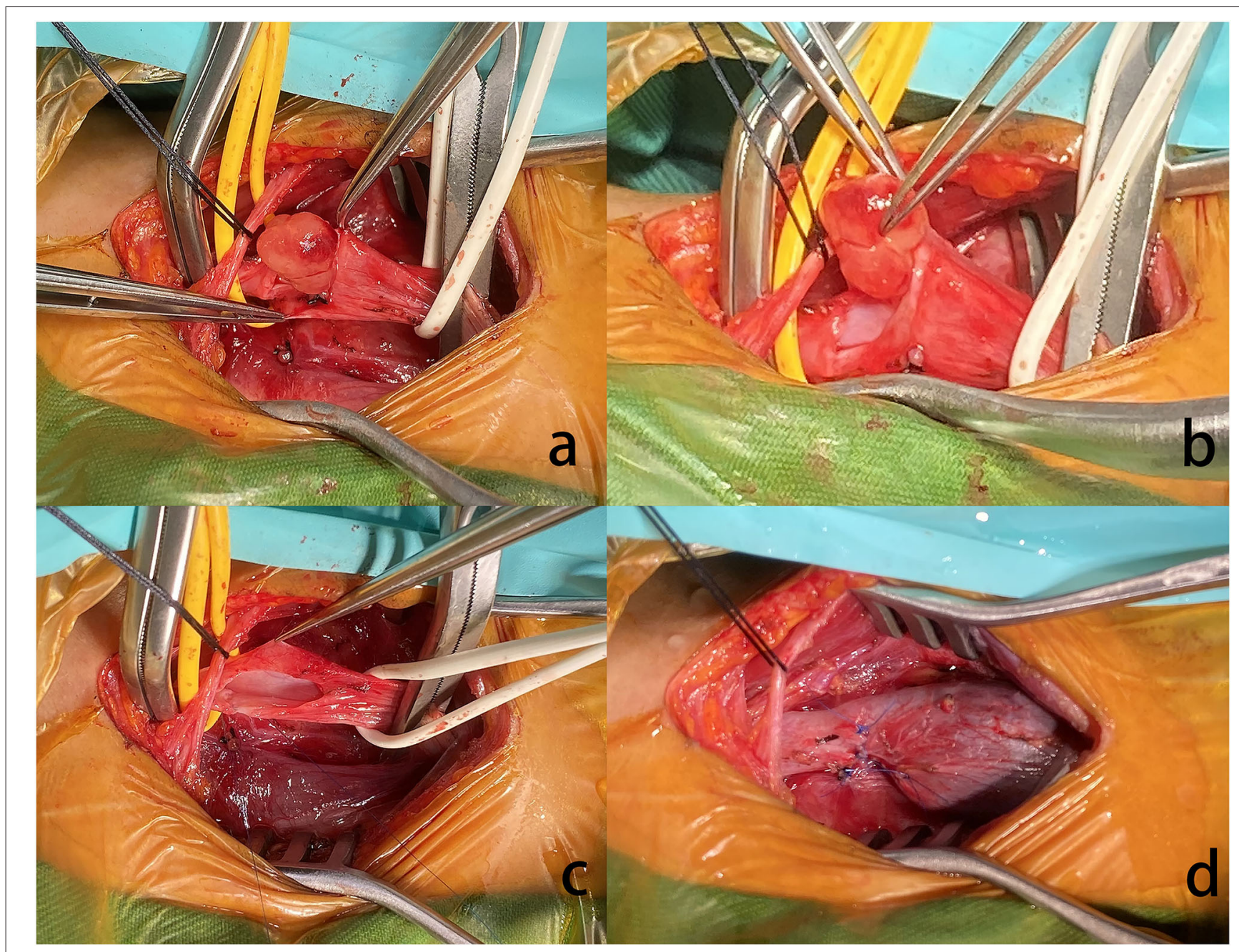

FIGURE 2 | (a) The nodule arose from the RJV. (b) Grossly, the mass was pink hyaline, oval, and well-demarcated, measuring 5 mm in diameter. (c) Extravascular tissues were not affected. (d) Due to the proximal of RJV compensatory dilation, local stenosis was observed in the RJV after reconstruction.

(Table 1) (1-26). So far, IVF occurring in large veins is reported in five cases $(17,18,21,24,25)$. To our knowledge, there are no reports of intravascular fasciitis that has invaded the jugular vein as seen in this case. Possible predisposing factors of IVF are reported, including preexisting trauma, viral infection, pregnancy-related estrogen changes, and thrombosis, but the exact mechanism and causative factors remain unknown.

It can develop in almost any site and is most commonly encountered in the head and neck, followed by the upper extremities, lower extremities, and trunk $(1,18)$. There is no significant difference in the incidence of IVF between genders $(5,13,19)$. Compared with ordinary NF, IVF was originally reported to affect the adolescent and young adult population. Most patients are between the ages of 20 and 40 years (17, 19). The mass can present as single or multinodular (17). Clinical symptoms usually manifest as a solitary nodule located subcutaneously or within muscular tissue. The size of the lesions range from 0.6 to $5 \mathrm{~cm}$, and the course of the disease was 2 weeks -8 years (16). Other features manifested as with or without pain/tenderness, slow growing, mobility, and regional discomfort (20). Cases affecting the large veins often present symptoms of venous thrombosis. IVF is always misdiagnosed with thrombosis as was the case in our patient. There are no established imaging criteria for an accurate diagnosis of IVF. It is possible that high-resolution ultrasound accompanied by sensitive blood flow signals may distinguish the lesion from the thrombosis. The gross appearance is a non-encapsulated nodular mass with a firm-to-soft or gelatinous consistency. They may appear poorly circumscribed, and infiltrating masses and vascular invasion may be recognized. When the lesion was predominantly intravascular growth, it can form a serpentine or plexiform configuration (13).

Microscopically, IVF is mainly composed of a proliferation of plump spindle cells inside the lumen or associated with the wall of arteries and veins of all sizes, arranged in short 


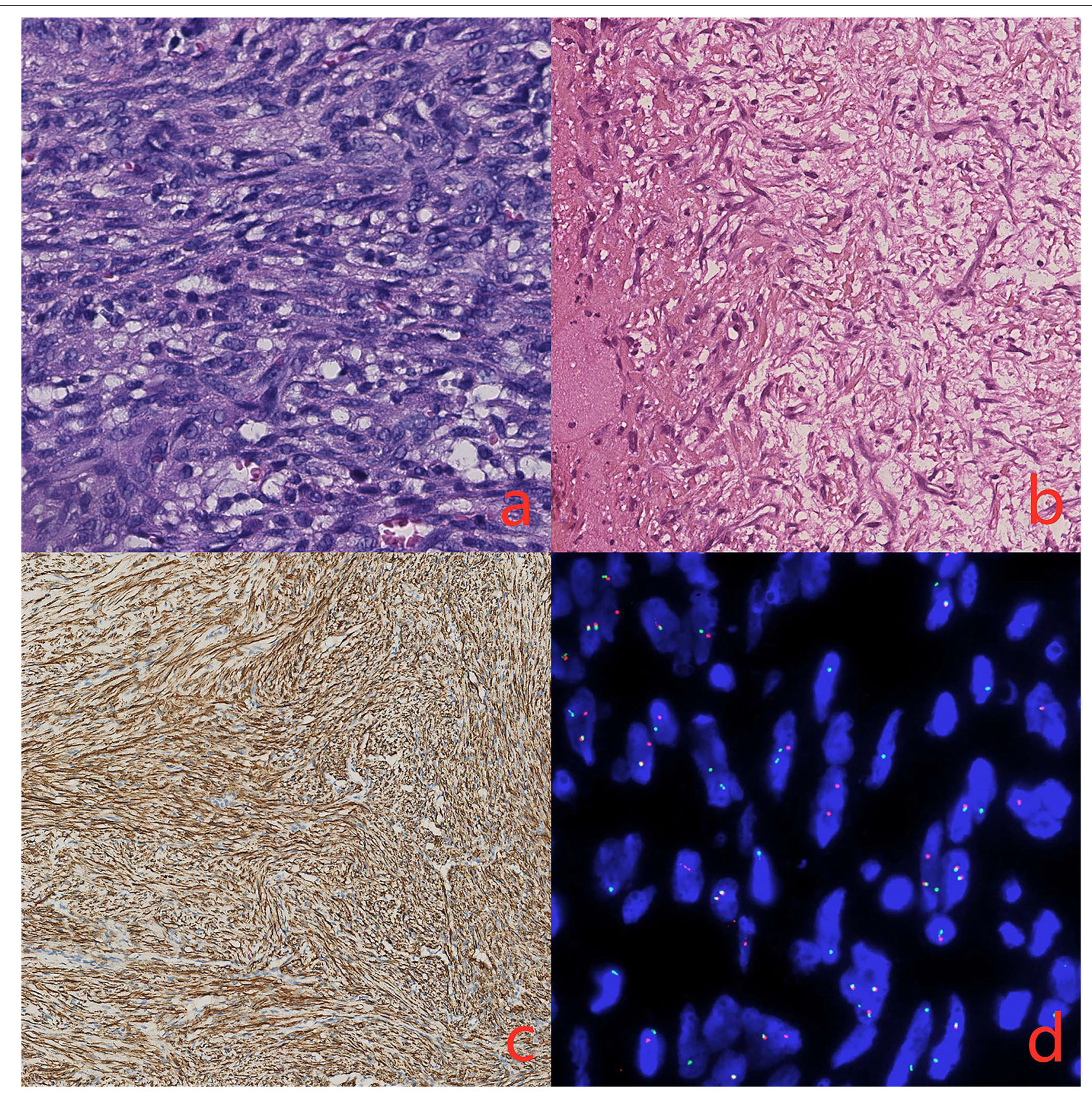

FIGURE 3 | (a) There is a proliferation of spindle cells arranged in intersecting fascicles (hematoxylin and eosin, original magnification 40x) (b). The

immunohistochemical stains demonstrated elastin stains was negative (original magnification 20x) (c). The spindle cells are diffusely positive for smooth muscle actin (SMA, original magnification 20x) (d). FISH showing rearrangement of the USP6 locus (separation of green and red signals).

intersecting fascicles, a storiform pattern, or a unregular manner. The lesions could appear as intraluminal or extraluminal growth with or without infiltration of surrounding connective tissue and infiltrative borders with entrapment of skeletal muscle observed in some cases. The background stroma was dense or myxoid and frequently showed a well-developed, slit-like capillary network. Scattered lymphocytes, red blood cells, and inflammatory cells often were seen in the background. Multinucleated giant cells were often present. Mitosis sometimes occurred, but no abnormal mitotic figures were found.

Immunohistochemistry studies often indicate that the spindle cells were positive for vimentin and SMA, negative for keratin, S100 protein, desmin, CD31, CD34, and c-kit, which confirmed the myofibroblastic differentiation seen in our patient $(13,19)$. 
TABLE 1 | Clinical and pathologic features of the reported cases of intravascular fasciitis.

\begin{tabular}{|c|c|c|c|c|c|c|c|c|c|}
\hline References & $\#$ & $\begin{array}{l}\text { Age (years) and } \\
\text { sex }\end{array}$ & Location & Symptoms & Gross features & $\begin{array}{l}\text { Microscopic } \\
\text { features }\end{array}$ & $\begin{array}{l}\text { IHC features of } \\
\text { spindle cells }\end{array}$ & Treatment & Follow-up \\
\hline $\begin{array}{l}\text { Patchefsky and } \\
\text { Enzinger (1) }\end{array}$ & 17 & $\begin{array}{l}20.5 \text { (range from } \\
0.5 \text { to } 57 \text { ). } \\
8 \mathrm{~F} \text { and } 9 \mathrm{M}\end{array}$ & $\begin{array}{l}\text { Head and neck } \\
(n=5) \\
\text { Upper extremity } \\
(n=7) \\
\text { Trunk } \\
(n=2) \\
\text { Lower extremity } \\
(n=3)\end{array}$ & No & $\begin{array}{l}1.5 \mathrm{~cm}^{\star} \text { (range } \\
\text { from } 0.6 \text { to } 5 \text { ) } \\
\text { single, firm, } \\
\text { immobile }\end{array}$ & $\begin{array}{l}\text { Feathery, } \\
\text { edematous } \\
\text { myxoid, and } \\
\text { hyalinized } \\
\text { background, giant } \\
\text { cells present in } 1 / 3 \\
\text { cases }\end{array}$ & N/A & $\mathrm{CR}$ & $\begin{array}{l}\text { Lost to follow-up } n=8 \\
\text { Alive and well } n=7 \\
\text { Local recurrence } n=2\end{array}$ \\
\hline \multirow[t]{2}{*}{$\begin{array}{l}\text { Freedman and } \\
\text { Lumerman (2) }\end{array}$} & 2 & $19, \mathrm{M}$ & $\begin{array}{l}\text { Right posterior } \\
\text { mucobuccal fold }\end{array}$ & No & $\begin{array}{l}2.5 \mathrm{~cm} \text {, single, } \\
\text { firm, with ulcer }\end{array}$ & $\begin{array}{l}\text { Myxoid and highly } \\
\text { vascular } \\
\text { background with } \\
\text { rare mitotic figures }\end{array}$ & N/A & $\mathrm{CR}$ & $N / A$ \\
\hline & & $53, \mathrm{M}$ & $\begin{array}{l}\text { Left buccal } \\
\text { mucosa }\end{array}$ & No & $\begin{array}{l}2.0 \mathrm{~cm} \text {, single, } \\
\text { firm, immobile } \\
\text { mass }\end{array}$ & $\begin{array}{l}\text { Myxoid and locally } \\
\text { hyalinized } \\
\text { background, no } \\
\text { mitotic figures } \\
\text { present }\end{array}$ & N/A & $\mathrm{CR}$ & $N / A$ \\
\hline Kahn et al. (3) & 1 & $20, \mathrm{~F}$ & $\begin{array}{l}\text { Left lower labial } \\
\text { mucosa }\end{array}$ & No & $\begin{array}{l}1.5 \mathrm{~cm} \text {, single, } \\
\text { firm, immobile }\end{array}$ & $\begin{array}{l}\text { Myxoid and highly } \\
\text { vascular } \\
\text { background, giant } \\
\text { cells and mitotic } \\
\text { figures present }\end{array}$ & N/A & $\mathrm{CR}$ & 3 months NRM \\
\hline \multirow[t]{2}{*}{ Price et al. (4) } & 2 & $17, \mathrm{M}$ & Right eye & Local swelling & $2.0 \mathrm{~cm}$, single & $\begin{array}{l}\text { Myxoid } \\
\text { background, giant } \\
\text { cells and mitotic } \\
\text { figures (<1/HPF) } \\
\text { present }\end{array}$ & $\mathrm{N} / \mathrm{A}$ & $\mathrm{CR}$ & 36 months NRM \\
\hline & & $20, M$ & Right eye & Local swelling & $\begin{array}{l}1.0 \mathrm{~cm} \text {, single, } \\
\text { firm, mobile }\end{array}$ & $\begin{array}{l}\text { Myxoid } \\
\text { background, giant } \\
\text { cells and mitotic } \\
\text { figures }(<1 / \text { HPF }) \\
\text { present }\end{array}$ & $\mathrm{N} / \mathrm{A}$ & $\mathrm{CR}$ & 36 months NRM \\
\hline $\begin{array}{l}\text { Samaratunga et al. } \\
\text { (5) }\end{array}$ & 1 & $49, \mathrm{M}$ & $\begin{array}{l}\text { Left inguinal } \\
\text { region }\end{array}$ & Local discomfort & $3.0 \mathrm{~cm}$, single, firm & $\begin{array}{l}\text { Myxoid } \\
\text { background with } \\
\text { cleft-like spaces, } \\
\text { giant cells and } \\
\text { mitotic figures } \\
\text { (2/10 HPF) present }\end{array}$ & $\begin{array}{l}\text { (+) Vimentin, SMA } \\
\text { (-) LMWK, S100 }\end{array}$ & $\mathrm{CR}$ & 6 months NRM \\
\hline Beer et al. (6) & 1 & $18, \mathrm{~F}$ & Lateral thigh & No & $\begin{array}{l}2.0 \mathrm{~cm} \text {, single, } \\
\text { tender, mobile }\end{array}$ & $\begin{array}{l}\text { Focally myxoid } \\
\text { and highly vascular } \\
\text { background, } \\
\text { mitosis present }\end{array}$ & $\begin{array}{l}\text { (+) SMA } \\
\text { (-) S100 }\end{array}$ & $\mathrm{CR}$ & 24 months NRM \\
\hline
\end{tabular}


TABLE 1 | Continued

\begin{tabular}{|c|c|c|c|c|c|c|c|c|c|}
\hline References & \# & $\begin{array}{l}\text { Age (years) and } \\
\text { sex }\end{array}$ & Location & Symptoms & Gross features & $\begin{array}{l}\text { Microscopic } \\
\text { features }\end{array}$ & $\begin{array}{l}\text { IHC features of } \\
\text { spindle cells }\end{array}$ & Treatment & Follow-up \\
\hline Sticha et al. (7) & 1 & $4, M$ & Right foot & No & $\begin{array}{l}3.0 \mathrm{~cm} \text {, single, } \\
\text { firm, tender, } \\
\text { immobile mass }\end{array}$ & $\begin{array}{l}\text { Myxomatous and } \\
\text { hyalinized } \\
\text { background, giant } \\
\text { cell and mitosis } \\
\text { present }\end{array}$ & $\mathrm{N} / \mathrm{A}$ & $\mathrm{CR}$ & 24 months NRM \\
\hline Ito et al. (8) & 1 & $26, M$ & Right forearm & Local tenderness & Single, tender & $\begin{array}{l}\text { Fibrous and } \\
\text { vascular } \\
\text { background, giant } \\
\text { cells and mitotic } \\
\text { figures (up to 1/10 } \\
\text { HPF) present }\end{array}$ & $\begin{array}{l}\text { (+) Vimentin, SMA } \\
\text { (-) Desmin }\end{array}$ & $\mathrm{CR}$ & N/A \\
\hline Anand et al. (9) & 1 & $20, F$ & Right hand & Local swelling & $\begin{array}{l}3.0 \mathrm{~cm} \text {, single, } \\
\text { firm, mobile }\end{array}$ & $\begin{array}{l}\text { Fibrous } \\
\text { background, giant } \\
\text { cells, and mitotic } \\
\text { figures present }\end{array}$ & $\begin{array}{l}\text { (+) SMA } \\
\text { (-) S100, desmin }\end{array}$ & $\mathrm{CR}$ & 12 months NRM \\
\hline $\begin{array}{l}\text { Sugaya and } \\
\text { Tamaki (10) }\end{array}$ & 1 & $66, M$ & $\begin{array}{l}\text { Medial border of } \\
\text { right foot }\end{array}$ & No & $\begin{array}{l}0.3 \mathrm{~cm} \text {, single, } \\
\text { mobile mass }\end{array}$ & $\begin{array}{l}\text { Myxoid } \\
\text { background, no } \\
\text { giant cells present, } \\
\text { rare mitotic figures }\end{array}$ & $\begin{array}{l}\text { (+) Vimentin } \\
\text { (-) S100, SMA, } \\
\text { c-ki, Desmin, CD3, } \\
\text { CD34, cytokeratin }\end{array}$ & $\mathrm{CR}$ & $\mathrm{N} / \mathrm{A}$ \\
\hline $\begin{array}{l}\text { Pantanowitz and } \\
\text { Duke (11) }\end{array}$ & 1 & $17, \mathrm{M}$ & Wrist & Pain swelling & $\begin{array}{l}1.2 \mathrm{~cm} \text {, single } \\
\text { mass }\end{array}$ & N/A & $\mathrm{N} / \mathrm{A}$ & $\mathrm{CR}$ & $12 \mathrm{~m}, \mathrm{NRM}$ \\
\hline Wang et al. (12) & 1 & $28, \mathrm{~F}$ & Left leg & No & $\begin{array}{l}\text { Multiple, firm, } \\
\text { masses }\end{array}$ & $\begin{array}{l}\text { Myxoid } \\
\text { background, no } \\
\text { giant cells or } \\
\text { mitotic figures } \\
\text { present }\end{array}$ & $\begin{array}{l}\text { (+) Vimentin, SMA } \\
\text { (-) Ketatin, } \\
\text { S100, desmin }\end{array}$ & $\mathrm{CR}$ & N/A \\
\hline Chi et al. (13) & 1 & $20, F$ & Upper lip & No & $\begin{array}{l}0.5 \mathrm{~cm} \text {, single, } \\
\text { firm, mobile }\end{array}$ & $\begin{array}{l}\text { Giant cells and } \\
\text { mitotic figures } \\
(11 / 10 \text { HPF) } \\
\text { present }\end{array}$ & $\begin{array}{l}\text { (+) SMA } \\
\text { (-) S100 }\end{array}$ & $\mathrm{CR}$ & 24 months NRM \\
\hline Reiser et al. (14) & 1 & $58, \mathrm{~F}$ & Right cheek & Local swelling & $1.7 \mathrm{~cm}$, single & $\begin{array}{l}\text { Focally myxoid } \\
\text { and highly vascular } \\
\text { background, no } \\
\text { giant cells present, } \\
\text { rare mitotic figures }\end{array}$ & $\begin{array}{l}\text { (+) SMA, } \\
(-) \text { S100, } \\
\text { Desmin, EMA }\end{array}$ & $\mathrm{CR}$ & 12 months NRM \\
\hline Seo et al. (15) & 1 & 26, M & Lower Lip & No & $1 \mathrm{~cm}$, single & $\begin{array}{l}\text { Fibrous } \\
\text { background, giant } \\
\text { cells present with } \\
\text { no mitotic figures }\end{array}$ & $\begin{array}{l}\text { (+) SMA } \\
\text { (-) CD31, CD34, } \\
\text { desmin, S-100 }\end{array}$ & $\mathrm{CR}$ & 2 months NRM \\
\hline Zheng et al. (16) & 1 & $21, F$ & Flank & No & $0.5 \mathrm{~cm}$, single & $\begin{array}{l}\text { Fibrous } \\
\text { background, giant } \\
\text { cells present with } \\
\text { no mitotic figures }\end{array}$ & $\begin{array}{l}\text { (+) SMA, FMSA } \\
(-) \text { S100 }\end{array}$ & $\mathrm{CR}$ & $\mathrm{N} / \mathrm{A}$ \\
\hline
\end{tabular}


TABLE 1 | Continued

\begin{tabular}{|c|c|c|c|c|c|c|c|c|c|}
\hline References & $\#$ & $\begin{array}{l}\text { Age (years) and } \\
\text { sex }\end{array}$ & Location & Symptoms & Gross features & $\begin{array}{l}\text { Microscopic } \\
\text { features }\end{array}$ & $\begin{array}{l}\text { IHC features of } \\
\text { spindle cells }\end{array}$ & Treatment & Follow-up \\
\hline Min et al. (17) & 1 & $29, F$ & $\begin{array}{l}\text { Left common } \\
\text { femoral vein }\end{array}$ & Pain swelling & $4.5 \mathrm{~cm}$, single & $\begin{array}{l}\text { Spindle cells with } \\
\text { mild nuclear atypia }\end{array}$ & $\begin{array}{l}\text { (+) SMA, CD34 } \\
\text { (-) Desmin, } \\
\text { Ki67 (7\%) }\end{array}$ & $\mathrm{CR}$ & 3 months NRM \\
\hline Lee et al. (18) & 1 & $41, \mathrm{~F}$ & $\begin{array}{l}\text { Left common } \\
\text { Femoral vein }\end{array}$ & Swelling & $3.8 \mathrm{~cm}$, single & $\begin{array}{l}\text { Fibrous } \\
\text { background, } \\
\text { present with no } \\
\text { mitotic figures. }\end{array}$ & $\begin{array}{l}\text { (+) SMA } \\
(-) \text { S100 }\end{array}$ & SRIV & $48 \mathrm{~m}, \mathrm{NRM}$ \\
\hline \multirow[t]{2}{*}{ Kuklani et al. (19) } & 2 & $25, \mathrm{~F}$ & Tongue and mouth & Swelling & Multiple, firm & $\begin{array}{l}\text { Focally myxoid } \\
\text { and highly vascular } \\
\text { background, no } \\
\text { giant cells present, } \\
\text { rare mitotic figures }\end{array}$ & $\begin{array}{l}\text { (+) SMA, MSA. } \\
\text { (-) C-kit, DOG1, } \\
\text { S100, GFAP } \\
\text { Desmin, } \\
\text { Ki-67 (<5\%) }\end{array}$ & $\mathrm{CR}$ & 12 months NRM \\
\hline & & $26, M$ & Tongue & No & $1.0 \mathrm{~cm}$, single & $\begin{array}{l}\text { Fibrous } \\
\text { background, } \\
\text { present with no } \\
\text { mitotic figures. }\end{array}$ & $\begin{array}{l}\text { (+) SMA, vimentin, } \\
\text { CD34 } \\
\text { (-) Desmin, S100. } \\
\text { Ki-67 (<10\%) }\end{array}$ & $\mathrm{CR}$ & 5 months NRM \\
\hline $\begin{array}{l}\text { Takahashi et al. } \\
\text { (20) }\end{array}$ & 1 & $30, \mathrm{~F}$ & $\begin{array}{l}\text { Right inguinal } \\
\text { region }\end{array}$ & Pain & $2.0 \mathrm{~cm}$, single & $\begin{array}{l}\text { Fibrous } \\
\text { background, } \\
\text { present with no } \\
\text { mitotic figures. }\end{array}$ & $\begin{array}{l}\text { (+) SMA, } \\
\text { (-) Desmin, S100 }\end{array}$ & $\mathrm{CR}$ & 11 months NRM \\
\hline Kang et al. (21) & 1 & $44, \mathrm{~F}$ & $\begin{array}{l}\text { Left clavicular } \\
\text { intravenous }\end{array}$ & Pain & $4.5 \mathrm{~cm}$, single & $\begin{array}{l}\text { Fibrous } \\
\text { background, giant } \\
\text { cells present with } \\
\text { no mitotic figures }\end{array}$ & $\begin{array}{l}\text { (+) SMA, } \\
(-) \text { S100 }\end{array}$ & $\mathrm{CR}$ & 3 months NRM \\
\hline $\begin{array}{l}\text { Bartu and Dundr } \\
\text { (22) }\end{array}$ & 1 & $61, \mathrm{~F}$ & Ascending aorta & No & Single & $\begin{array}{l}\text { Fibrous } \\
\text { background } \\
\text { present with no } \\
\text { mitotic figures. }\end{array}$ & $\begin{array}{l}\text { (-) SMA, desmin, } \\
\text { S100 CD34, } \\
\text { CD31, } \\
\text { Ki-67 > 25\% }\end{array}$ & $\mathrm{CR}$ & 12 months NRM \\
\hline $\begin{array}{l}\text { Ding and Jiang } \\
\text { (23) }\end{array}$ & 1 & $42, \mathrm{~F}$ & $\begin{array}{l}\text { Left common } \\
\text { femoral venous }\end{array}$ & Pain swelling & $1.5 \mathrm{~cm}$, single & $\mathrm{N} / \mathrm{A}$ & $\begin{array}{l}\text { (+) SMA, } \\
\text { (-) S100, MYH9- } \\
\text { USP6 rearrangement }\end{array}$ & $\mathrm{CR}$ & $\mathrm{N} / \mathrm{A}$ \\
\hline Le et al. (24) & 1 & $23, F$ & $\begin{array}{l}\text { Left common } \\
\text { femoral vein }\end{array}$ & Pain swelling & Single, tender & $\begin{array}{l}\text { Fibrous } \\
\text { background, giant } \\
\text { cells present with } \\
\text { no mitotic figures }\end{array}$ & $\begin{array}{l}\text { (+) SMA } \\
\text { (-) S100 }\end{array}$ & $\mathrm{CR}$ & 24 months NRM \\
\hline Li et al. (25) & 1 & $39, \mathrm{~F}$ & $\begin{array}{l}\text { Left common } \\
\text { femoral vein }\end{array}$ & Pain swelling & Single & $\begin{array}{l}\text { Myxoid } \\
\text { background no } \\
\text { giant cells present } \\
\text { rare mitotic figures }\end{array}$ & $\begin{array}{l}\text { (+) SMA } \\
(-) \text { S100, desmin, } \\
\text { FLI-1, ALK, CD34 }\end{array}$ & $\mathrm{CR}$ & 6 months, NRM \\
\hline Pan et al. (26) & 1 & $27, M$ & $\begin{array}{l}\text { Left common } \\
\text { femoral vein }\end{array}$ & Pain swelling & $4.0 \mathrm{~cm}$, Single & N/A & $\begin{array}{l}\text { (+) SMA, vimentin } \\
\text { (-) Desmin, S100 }\end{array}$ & $\mathrm{CR}$ & 14 months, NRM \\
\hline
\end{tabular}

Size in greatest dimension

EMA, epithelial membrane antigen; HPF, high power field; LMWK, low molecular weight keratin; N/A, not applicable; SMA, smooth muscle actin; MSA, muscle-specific actin; FMSA, focal muscle specific actin; CR, complete resection; SRIV, Segmental excision of involved vein; NRM, no recurrence and metastasis; FLI-1. Friend leukemia virus integration-1. 
A fusion gene (MYH9-USP6) caused by the chromosomal translocation $t(17,22)$ was detected in our case. The fusion gene (MYH9-USP6) was detected in 92\% of NF and found to be highly sensitive and specific, favoring a neoplastic nature (27).

Due to the feature of infiltrative growth similar to a sarcoma (16), IVF also needs to be added to the differential diagnoses such as leiomyomatosis, hemangioendothelioma, fibroblastoma, myofibroblastoma, and leiomyosarcoma. Combined with the epidemiology, clinical symptoms, gross appearance, and microscopic and immunohistochemistry of IVF, it is not difficult to distinguish it from other tumors, especially microscopically and with immunohistochemistry. Surgical resection is the main method to treat IVF, and perioperative management is the guarantee of good surgical efficacy. It is very important to fully evaluate the nodule before surgery, including CTA and ultrasound contrast. According to current literature reports, there is no preoperative pathological puncture for IVF. The nodules should be completely removed during the operation, including the invaded vascular wall. As to whether the invaded vasculature needs to be reconstructed, it needs to be comprehensively evaluated based on the diameter, location, and scope of the invaded vasculature. Patients that are revascularized should be anticoagulated after surgery to avoid thrombosis. In general, surgical resection is the main treatment of IVF and the reoccurrence rate of IVF is $<1-2 \%$ $(12,13)$.

\section{REFERENCES}

1. Patchefsky AS, Enzinger FM. Intravascular fasciitis: a report of 17 cases. Am J Surg Pathol. (1981) 5:29-36. doi: 10.1097/00000478-198101000-00006

2. Freedman PD, Lumerman H. Intravascular fasciitis: report of two cases and review of the literature. Oral Surg Oral Med Oral Pathol. (1986) 62:549-54. doi: 10.1016/0030-4220(86)90 319-1

3. Kahn MA, Weathers DR, Johnson DM. Intravascular fasciitis: a case report of an intraoral location. J Oral Pathol. (1987) 16:303-6. doi: 10.1111/j.1600-0714.1987.tb00698.x

4. Price SK, Kahn LB, Saxe N. Dermal and intravascular fasciitis. Unusual variants of nodular fasciitis. Am J Dermatopathol. (1993) 15:539-43. doi: 10.1097/00000372-199312000-00004

5. Samaratunga $\mathrm{H}$, Searle J, O'Loughlin $\mathrm{B}$. Intravascular fasciitis: a case report and review of the literature. Pathology. (1996) 28:8-11. doi: 10.1080/00313029600169413

6. Beer K, Katz S, Medenica M. Intravascular fasciitis. Int J Dermatol. (1996) 35:147-8. doi: 10.1111/j.1365-4362.1996.tb03286.x

7. Sticha RS, Deacon JS, Wertheimer SJ, Danforth RD Jr. Intravascular fasciitis in the foot. $J$ Foot Ankle Surg. (1997) 36:95-9. doi: 10.1016/S1067-2516(97)80052-X

8. Ito M, Matsunaga K, Sano K, Sakaguchi N, Hotchi M. Intravascular fasciitis of the forearm vein: a case report with immunohistochemical characterization. Pathol Int. (1999) 49:175-9. doi: 10.1046/j.1440-1827.1999.00842.x

9. Anand A, Tsapakis EM, Narvani AA, Alhakim A, Cannon SR, Tsiridis E. "Pseudosarcoma” in a pregnant woman. World J Surg Oncol. (2007) 5:7. doi: 10.1186/1477-7819-5-7

10. Sugaya M, Tamaki K. Does thrombosis cause intravascular fasciitis? Acta Derm Venereol. (2007) 87:369-70. doi: 10.2340/00015555-0268

11. Pantanowitz L, Duke WH. Intravascular lesions of the hand. Diagn Pathol. (2008) 3:24. doi: 10.1186/1746-1596-3-24

\section{CONCLUSION}

IVF arising in the jugular vein is extremely uncommon. IVF is very easily misdiagnosed as sarcoma, thrombosis, or other low-grade malignant mesenchymal neoplasms. Combined with the epidemiology, clinical symptoms, gross appearance, microscopic and immunohistochemistry of IVF, it is not difficult to distinguish it from other tumors, especially microscopically and with immunohistochemistry.

\section{DATA AVAILABILITY STATEMENT}

The original contributions presented in the study are included in the article/supplementary material, further inquiries can be directed to the corresponding authors.

\section{ETHICS STATEMENT}

Written informed consent was obtained from the individual(s) for the publication of any potentially identifiable images or data included in this article.

\section{AUTHOR CONTRIBUTIONS}

GC and CC were wrote the manuscript and were assistant in surgery. XW was involved in editing the manuscript. $\mathrm{BH}$ was chief operating surgeon. All authors contributed to the article and approved the submitted version.

12. Wang L, Wang G, Wang L, Gao T. Myxoid intravascular fasciitis. J Cutan Pathol. (2011) 38:63-6. doi: 10.1111/j.1600-0560.2009.01445.x

13. Chi AC, Dunlap WS, Richardson MS, Neville BW. Intravascular fasciitis: report of an intraoral case and review of the literature. Head Neck Pathol. (2012) 6:140-5. doi: 10.1007/s12105-011-0284-9

14. Reiser V, Alterman M, Shlomi B, Issakov J, Dagan Y, Kleinman S, et al. Oral intravascular fasciitis: a rare maxillofacial lesion. Oral Surg Oral Med Oral Pathol Oral Radiol. (2012) 114:e40-4. doi: 10.1016/j.oooo.2012.03.027

15. Seo BF, Kim DJ, Kim SW, Lee JH, Rhie JW, Ahn ST. Intravascular fasciitis of the lower lip. J Craniofac Surg. (2013) 24:892-5. doi: 10.1097/SCS.0b013e3182801354

16. Zheng $\mathrm{Y}$, George $\mathrm{M}$, Chen F. Intravascular fasciitis involving the flank of a 21-year-old female: a case report and review of the literature. BMC Res Notes. (2014) 7:118. doi: 10.1186/1756-0500-7-118

17. Min SI, Han A, Choi C, Min SK, Ha J, Jung IM. Iliofemoral vein thrombosis due to an intravascular fasciitis. J Vasc Surg Cases. (2015) 1:7376. doi: 10.1016/j.jvsc.2014.10.003

18. Lee HG, Pyo JY, Park YW, Ro JY. Intravascular fasciitis of the common femoral vein. Vasa. (2015) 44:395-8. doi: 10.1024/0301-1526/a000460

19. Kuklani R, Robbins JL, Chalk EC, Pringle G. Intravascular fasciitis: report of two intraoral cases and review of the literature. Oral Surg Oral Med Oral Pathol Oral Radiol. (2016) 121:e19-25. doi: 10.1016/j.oooo.2015.05.014

20. Takahashi K, Yanagi T, Imafuku K, Kitamura S, Inamura-Takashima Y, Yamaguchi $\mathrm{Y}$, et al. Ultrasonographic features of intravascular fasciitis: case report and review of the literature. J Eur Acad Dermatol Venereol. (2017) 31:e457-9. doi: 10.1111/jdv.14293

21. Kang JH, Kim DI, Chung BH, Heo SH, Park YJ. A case report of the intravascular fasciitis of a neck vein mimicking intravascular tumorous conditions. Ann Vasc Dis. (2018) 11:553-6. doi: 10.3400/avd.cr.18-00065

22. Bartu M, Dundr P, Nemejcova K, Prokopova P, Zambo I, Cerny S. Intravascular fasciitis leading to an aortic dissection. A case report. Cesk Patol. (2018) 63:196-9. 
23. Ding $\mathrm{X}$, Jiang J. Intravascular fasciitis of the femoral vein mimicking thrombosis and sarcoma. Eur J Vasc Endovasc Surg. (2020) 61:373. doi: 10.1016/j.ejvs.2020.10.012

24. Le P, Servais AB, Salehi P. Intravascular fasciitis presenting as recurrent deep venous thrombosis. J Vasc Surg Cases Innov Tech. (2020) 6:60911. doi: 10.1016/j.jvscit.2020.08.026

25. Li N, Hong DK, Zheng XX, Zhou YD, Chen XS. Images in vascular medicine: intravascular fasciitis of the common femoral vein mimicking deep venous thrombosis. Vasc Med. (2020) 25:602-3. doi: 10.1177/1358863X20938125

26. Pan H, Zhou L, Deng C, Zheng J, Chen K, Gao Z. A rare case of intravascular fasciitis misdiagnosed as deep venous thrombosis. Ann Vasc Surg. (2020) 62:499 e495-8. doi: 10.1016/j.avsg.2019.0 7.005

27. Lu Y, He X, Qiu Y, Chen $H$, Zhuang $H$, Yao J, et al. Novel CTNNB1-USP6 fusion in intravascular fasciitis of the large vein identified by next-generation sequencing. Virchows Arch. (2020) 477:455-9. doi: 10.1007/s00428-020-02792-x
Conflict of Interest: The authors declare that the research was conducted in the absence of any commercial or financial relationships that could be construed as a potential conflict of interest.

Publisher's Note: All claims expressed in this article are solely those of the authors and do not necessarily represent those of their affiliated organizations, or those of the publisher, the editors and the reviewers. Any product that may be evaluated in this article, or claim that may be made by its manufacturer, is not guaranteed or endorsed by the publisher.

Copyright (c) 2021 Chen, Chen, Wen and Huang. This is an open-access article distributed under the terms of the Creative Commons Attribution License (CC BY). The use, distribution or reproduction in other forums is permitted, provided the original author(s) and the copyright owner(s) are credited and that the original publication in this journal is cited, in accordance with accepted academic practice. No use, distribution or reproduction is permitted which does not comply with these terms. 